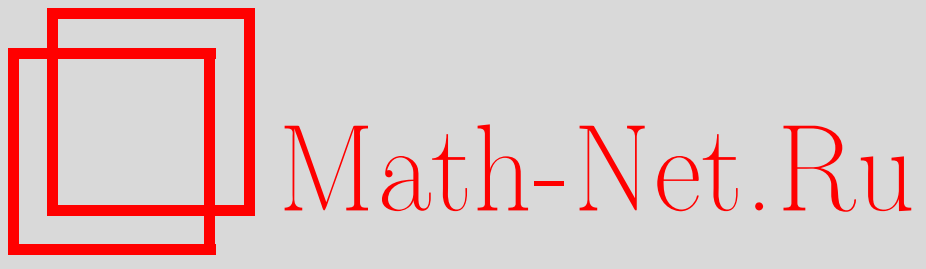

С. В. Шадрин, Полиномиальные числа Гурвица и пересечения на $\overline{\mathcal{M}}_{0, k}$, Функи. анализ и его прил., 2003, том 37, выпуск 1, 92-94

DOI: https://doi.org/10.4213/faa142

Использование Общероссийского математического портала MathNet.Ru подразумевает, что вы прочитали и согласны с пользовательским соглашением

http://www . mathnet.ru/rus/agreement

Параметры загрузки:

IP : 54.224 .135 .184

26 апреля 2023 г., 13:58:32

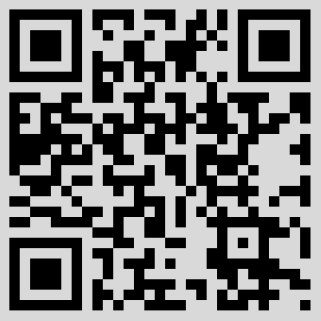


УДК 512.772 .5

\title{
Полиномиальные числа Гурвица и пересечения на $\overline{\mathscr{M}}_{0, k}{ }^{*}$
}

\author{
(c) 2003. С. В. ШАдРин
}

1. Рассмотрим многочлен $P$ степени $n$. Пусть $\Sigma=\left\{x_{1}, \ldots, x_{m}\right\}-$ конечное подмножество в $\mathbb{C P}^{1}$, содержащее все критические значения многочлена $P$, в том числе и бесконечность (будем считать, что $x_{1}=\infty$ ). Паспортом точки $x_{i} \in \Sigma$ (относительно многочлена $P$ ) называется набор натуральных чисел $A_{i}(P)=\left(a_{1}^{i}, \ldots, a_{l_{i}}^{i}\right)$, такой, что $P^{-1}\left(x_{i}\right)=a_{1}^{i} y_{1}^{i}+\cdots+a_{l_{i}}^{i} y_{l_{i}}^{i}$, где $y_{1}^{i}, \ldots, y_{l_{i}}^{i}-$ различные точки сферы $\mathbb{C P}^{1}$.

В частности, паспорт $A_{1}(P)$ точки $x_{1}=\infty$ равен $(n)$. Если точка $x_{i}-$ peгулярное значение многочлена $P$, то $A_{i}(P)=(1, \ldots, 1)$. Если $x_{i}-$ простое критическое значение многочлена $P$, то $A_{i}(P)=(2,1, \ldots, 1)$.

2. Припишем каждой точке $x_{i} \in \Sigma$ какой-нибудь паспорт $A_{i}=\left(a_{1}^{i}, \ldots, a_{l_{i}}^{i}\right)$. Пусть $A_{1}=(n), \sum_{j=1}^{l_{i}} a_{j}^{i}=n$ для любого $i$ и $\sum_{i=1}^{m}\left(n-l_{i}\right)=2 n-2$. Эти условия необходимы для существования многочлена $P$, все критические значения которого лежат в $\Sigma$, такого, что $A_{i}(P)=A_{i}$ для любого $i=1, \ldots, m$.

Числом Гурвица $h$ называется число таких многочленов $P$. При этом мы рассматриваем многочлены с точностью до действия группы $\mathrm{PGL}(2, \mathbb{C})$ в прообразе; каждый многочлен учитывается с весом $1 / c$, где $c$ - число автоморфизмов многочлена.

Для этого числа в [5] найдена несложная комбинаторная формула. Мы же хотим выразить это число через числа пересечений на $\overline{\mathscr{M}}_{0, k}$. Для задачи вычисления чисел Гурвица наша формула не даст ничего нового, но подобные формулы имеют самостоятельный интерес и бывают очень полезны (см. $[1,6])$.

3. Через $\overline{\mathscr{M}}_{0, k}$ обозначается пространство стабильных кривых рода 0 с $k$ отмеченными точками. Через $\psi(y)$ обозначается первый класс Черна расслоения над $\overline{\mathscr{M}}_{0, k}$ со слоем, равным кокасательной прямой в отмеченной точке $y$.

Мы будем рассматривать пересечения на пространстве $\overline{\mathscr{M}}_{0, \sum_{i=1}^{m} l_{i}}$, причем отмеченные точки обозначим через $y_{j}^{i}, i=1, \ldots, m, j=1, \ldots, l_{i}$.

Обозначим через $\pi_{p, q, k}: \overline{\mathscr{M}}_{0, \sum_{i=1}^{m} l_{m}} \rightarrow \overline{\mathscr{M}}_{2+l_{p}}$ проекцию, забывающую все отмеченные точки, кроме $y_{1}^{1}, y_{1}^{p}, \ldots, y_{l_{p}}^{p}$ и $y_{k}^{q}$. Обозначим через $D_{U}, U \subset\left\{y_{j}^{i}\right\}$, класс когомологий, двойственный дивизору, общая точка которого представлена двухкомпонентной кривой, такой, что все точки из множества $U$ лежат на одной компоненте, а все точки из множества $\left\{y_{j}^{i}\right\} \backslash U-$ на другой компоненте.

Определим классы $\Psi_{p}\left(b_{j}^{i}\right)_{j=1, \ldots, l_{i}}^{i=2, \ldots, p+1, \ldots, m}$ с помощью рекуррентных соотношений. Если $\left(b_{j}^{i}\right)=0$, то $\Psi_{p}\left(b_{j}^{i}\right)=1$. Пусть набор числе $\widehat{b}_{j}^{i}$ отличается от $b_{j}^{i}$ только при $i=q, j=k$, где $\widehat{b}_{k}^{q}=b_{k}^{q}+1$. Тогда

$$
\Psi_{p}\left(\widehat{b}_{j}^{i}\right)=\widehat{b}_{k}^{q} \pi_{p, q, k}^{*} \psi\left(y_{k}^{q}\right) \Psi_{p}\left(b_{j}^{i}\right)-\sum_{U} a_{U} \Psi_{p}\left(\left(b_{U}\right)_{j}^{i}\right) D_{U \cup\left\{y_{k}^{q}\right\}},
$$

*Работа частично поддержана грантами РФФИ-01-01-00660 и INTAS-00-0259. 
где суммирование ведется по всем $U \subset\left\{y_{j}^{i}\right\} \backslash\left\{y_{1}^{1}, y_{1}^{p}, \ldots, y_{l_{p}}^{p}, y_{k}^{q}\right\}, a_{U}=\sum_{y_{j}^{i} \in U} b_{j}^{i}$, $\left(b_{U}\right)_{j}^{i}=b_{j}^{i}$, если $y_{j}^{i} \notin U \cup\left\{y_{k}^{q}\right\}$, и $\left(b_{U}\right)_{j}^{i}=0$, если $y_{j}^{i} \in U$, а $\left(b_{U}\right)_{k}^{q}=a_{U}+b_{k}^{q}$. Корректность такого определения не вполне тривиально следует из теоремы Кила [4]. Мы будем использовать лишь классы $\Psi_{p}=\Psi_{p}\left(a_{j}^{i}-1\right)$, т. е. есть классы, определенные по набору чисел $\left(a_{j}^{i}-1\right)_{j=1, \ldots, l_{i}}^{i=2, \ldots, p-1, p+1, \ldots, m}$.

4. Сформулируем теорему.

Теорема. Пусть $m \geqslant 3$. Тогда имеет место следующая формула:

$$
h=\frac{n^{m-3}}{\prod_{i=1}^{m} \operatorname{aut}\left(A_{i}\right)} \int_{\overline{\mathscr{M}}_{0, \sum_{i=1}^{m} l_{m}}} \psi\left(y_{1}^{1}\right)^{m-3} \prod_{p=2}^{m} \Psi_{p} .
$$

5. Покажем, как можно проверить, что эта формула верна в простейших случаях.

Случай 1. Пусть $m=n, A_{1}=(n), A_{2}=\cdots=A_{m}=(2,1, \ldots, 1)$. Иначе говоря, мы рассматриваем случай, когда все критические значения простые.

Можно доказать, что $\Psi_{p}$ в этом случае двойствен подмногообразию, общая точка которого состоит из $(n-1)$-компонентной кривой, причем $y_{1}^{1}$ и $y_{1}^{p}$ лежат на одной компоненте, с которой пересекаются оставшиеся $n-2$ компонент, а на каждой из этих компонент лежит ровно одна из точек $y_{1}^{i}$ и ровно одна из точек $y_{j}^{p}$.

$\mathrm{У}$ каждого такого подмногообразия имеется $n-2$ неприводимых компонент. Соответственно интегральная часть формулы равна $(n-2) !^{n-1}$, и $h=n^{n-3}$.

Случай 2. Пусть $m=3, A_{1}=(n), A_{2}=\left(a_{1}^{2}, a_{2}^{2}\right), A_{3}=(2,1, \ldots, 1)$. Тогда мы имеем

$$
\begin{aligned}
\Psi_{2}= & \pi_{2,3,1}^{*} \psi\left(y_{1}^{3}\right), \\
\Psi_{3}= & \left(a_{1}^{2}-1\right) !\left(a_{2}^{2}-1\right) ! \pi_{3,2,1}^{*} \psi\left(y_{1}^{2}\right)^{a_{1}^{2}-1} \pi_{3,2,2}^{*} \psi\left(y_{2}^{2}\right)^{a_{2}^{2}-1} \\
& \quad-\left((n-2) !-\left(a_{1}^{2}-1\right) !\left(a_{2}^{2}-1\right) !\right) D_{\left\{y_{1}^{2}, y_{2}^{2}\right\}} \pi_{3,2,1}^{*} \psi\left(y_{1}^{2}\right)^{n-3} .
\end{aligned}
$$

Заметим, что $\Psi_{2}$ двойствен дивизору, общая точка которого представлена двухкомпонентной кривой, такой, что $y_{1}^{1}$ и $y_{1}^{2}$ лежат на одной компоненте, а $y_{2}^{2}$ и $y_{1}^{3}-$ на другой. Ограничение класса $\Psi_{3}$ на этот дивизор равно

$$
\left(a_{1}^{2}-1\right) !\left(a_{2}^{2}-1\right) ! \psi\left(y_{1}^{2}\right)^{a_{1}^{2}-1} \psi\left(y_{2}^{2}\right)^{a_{2}^{2}-1} .
$$

Далее, стандартными вычислениями [4] мы получаем, что $h=1 / \operatorname{aut}\left(A_{2}\right)$.

6. Изложим схему доказательства теоремы.

Рассмотрим $\Sigma$ как точку из $\mathscr{M}_{0, m}$. Соответственно вместо многочленов мы рассмотрим пары $(P, \Sigma)$, где $P-$ многочлен, $\Sigma \in \mathscr{M}_{0, m}$ и все паспорта фиксированны.

Отображение $l l:(P, \Sigma) \mapsto \Sigma-$ это конечнолистное накрытие над $\mathscr{M}_{0, m}$, а число $h-$ кратность этого отбражения.

Существует компактификация Харриса-Мамфорда $\bar{H}$ пространства пар $(P, \Sigma)$ (см. [2]), такая, что отображение $l l$ продолжается до разветвленного накрытия $l l: \bar{H} \rightarrow \overline{\mathscr{M}}_{0, m}$.

Тогда, так как $\int_{\mathscr{M}_{0, m}} \psi\left(x_{1}\right)^{m-3}=1$, то $h=\int_{\bar{H}}\left(l l^{*} \psi\left(x_{1}\right)\right)^{m-3}$. 
Пронумеруем все прообразы всех точек из $\Sigma$. Рассмотрим отображение $s t: \bar{H} \rightarrow$ $\overline{\mathscr{M}}_{0, \sum_{i=1}^{m} l_{i}}$, сопоставляющее точке $(P, \Sigma)$ кривую-прообраз многочлена $P$ с отмеченными всеми прообразами всех точек из $\Sigma$.

Согласно лемме из [3], $n \cdot s t^{*} \psi\left(y_{1}^{1}\right)=l l^{*} \psi\left(x_{1}\right)$ (если $y_{1}^{1}-$ это прообраз точки $x_{1}$ кратности $\left.n\right)$. Следовательно, $h=\int_{\bar{H}}\left(n \cdot s t^{*} \psi\left(y_{1}^{1}\right)^{m-3} /\left(\prod_{i=1}^{m} \operatorname{aut}\left(A_{i}\right)\right)\right.$ (здесь мы еще делим на количество возможных нумераций всех прообразов всех точек из $\Sigma)$.

Для получения формулы (2) осталось только вычислить класс $s t_{*} \bar{H}$. Это можно сделать, правильно интерпретировав в терминах пересечений условия, что некоторые отмеченные точки являются критическими точками некоторого многочлена с заданными порядками ветвления и что значения этого многочлена в некоторых отмеченных точках совпадают.

7. Автор выражает благодарность С. К. Ландо, С. М. Натанзону и М. З. Шапиро за полезные обсуждения. Полное доказательство теоремы см. в [7].

\section{ЛИТЕРАТУРА}

1. Ekedahl T., Lando S., Shapiro M., Vainshtein A. Invent. Math., 146, 297-327 (2001). 2. Harris J., Mumford D. Invent. Math., 67, 23-86 (1982). 3. Ionel E. Invent. Math., 148, No. 3, 627-658 (2002). 4. Keel S. Trans. Amer. Math. Soc., 330, No. 2, 545-574 (1992). 5. Ландо С. К., Звонкин Д. А. Функц. анализ и его прил., 33, вып. 3, 21-34 (1999). 6. Okounkov A., Pandharipande R. arXiv: math.AG/0101147. 7. Shadrin S. V. arXiv: math.AG/0209282.

Московский государственный университет им. М. В. Ломоносова

Поступило в редакцию Независимый московский университет e-mail: shadrin@mccme.ru 